\title{
Medical Image of the Week: Dobhoff Placement in a Patient with Hiatal Hernia
}

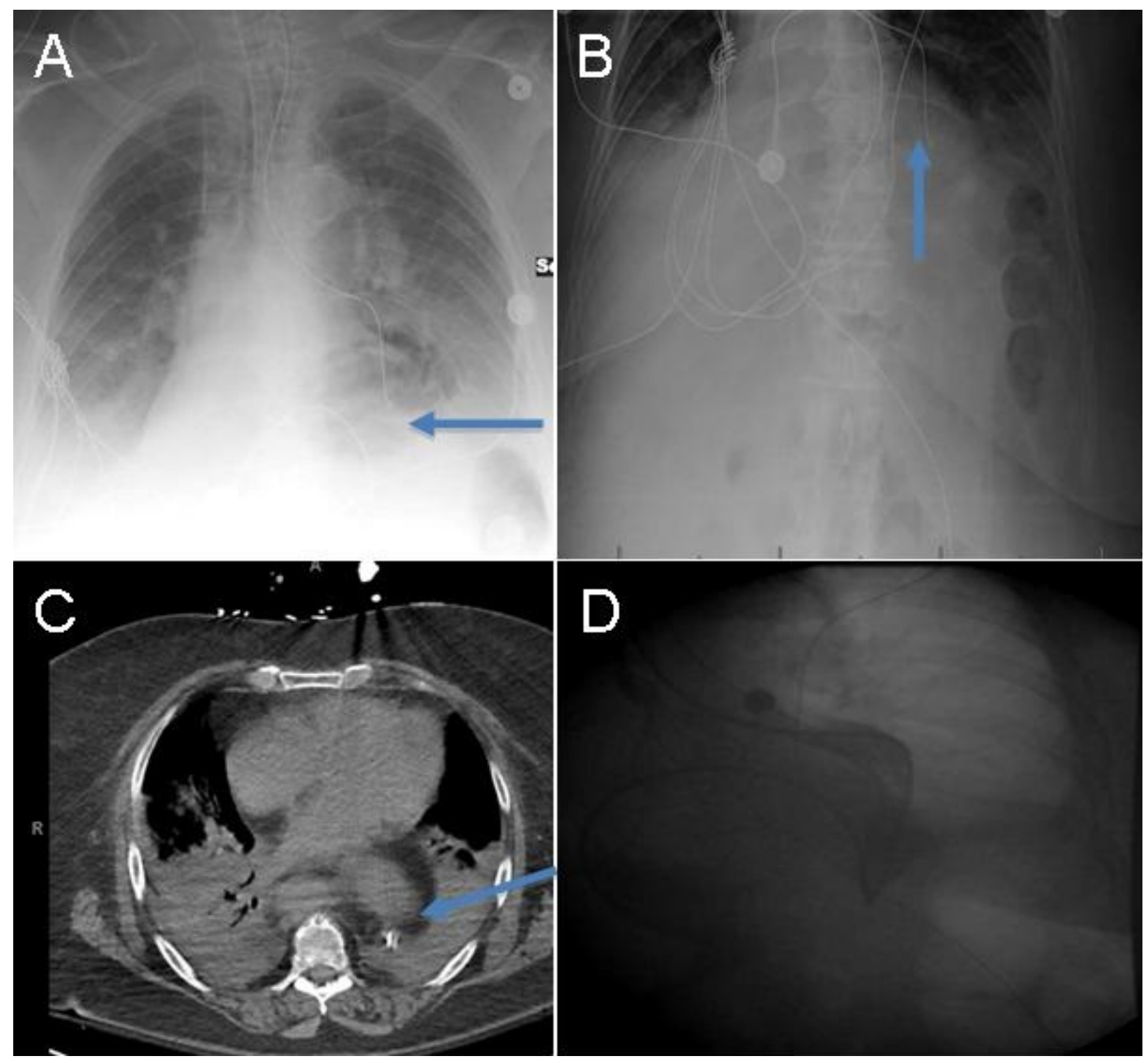

Figure 1. Arrows designate tip of Dobhoff feeding tube (DHT). Panel A: Chest radiograph. DHT appears to follow the left main bronchus into the left lower lobe. Panel B: Abdominal view of DHT placement. Panel C: Chest CT showing degree of hiatal hernia and DHT in the intra-thoracic hernia. Panel D: Follow-up fluoroscopy imaging showing appropriately placed DHT in the duodenum.

A 79 year-old woman with a past medical history of obstructive sleep apnea, chronic obstructive pulmonary disease on home oxygen, obesity hypoventilation syndrome, hypertension, and anxiety presented with a 2 day history of altered mental status and symptoms consistent with a COPD exacerbation, including dyspnea and increased oxygen requirements. She was found to be hypercarbic and did not tolerate a trial of BiPAP due to her altered mentation. She was subsequently intubated. Due to an 
expected prolonged intubation period, plans for enteral access were made. A Dobhoff naso-duodenal feeding tube (DHT) was inserted. On chest radiograph and a concurrent abdominal radiograph, the DHT appeared to have been inserted into the left mainstem bronchus terminating in the left lower lobe (Figure 1A and 1B). The nursing staff removed and replaced the DHT resulting in a similar radiograph. A third placement was attempted with similar radiographic results. Therefore, a computed tomography (CT) scan of the chest was performed to evaluate tube placement. The CT of the chest showed a large hiatal hernia contained within thoracic cavity (Figure 1C). Upon chart review, previous radiographs mentioned hiatal hernia but it appeared that the degree of herniation had progressed. Fluoroscopy was used to confirm placement of the DHT beyond the herniated gastric contents into the duodenum (Figure 1D) and tube feeds were initiated.

Post-pyloric feeding tubes are often used in place of gastric feeding tubes under the assumption that the risk of aspiration in the intubated patient is reduced. Enteral nutrition is typically started within 36 hours of intubation as this has been shown to decrease mortality in intubated patients (1). There are contraindications to the use of nasogastric or nasoenteric feeding tubes, which include facial trauma, esophageal web, or recent esophagectomy. Hiatal hernias are not a contraindication to nasoenteric feeding tube placement, however, patients with unusual anatomy may benefit from placement under fluoroscopic or endoscopic visualization in order to ensure appropriate positioning (2).

Kawanjit K Sekhon, MD and Ryan Nahapetian, MD, MPH

Department of Internal Medicine

Division of Pulmonary, Allergy, Critical Care, and Sleep Medicine

University of Arizona, Tucson, AZ

\section{References}

1. Marik PE, Zaloga GP. Gastric versus post-pyloric feeding: a systematic review. Crit Care. 2003;7(3):R46-51. [CrossRef] [PubMed]

2. Hodin RA, Bordeianou L. Nasogastric and nasoenteric tubes. Uptodate.com. Oct 17, 2013. Dec 20, 2013. Available at: http://www.uptodate.com/contents/nasogastric-andnasoenterictubes?source=machineLearning \&search=hiatal+hernia+feeding+tube\&selectedTitle $=1 \%$ 7E150\&sectionRank=3\&anchor=H522922014\#H522922014 (requires subscription). 